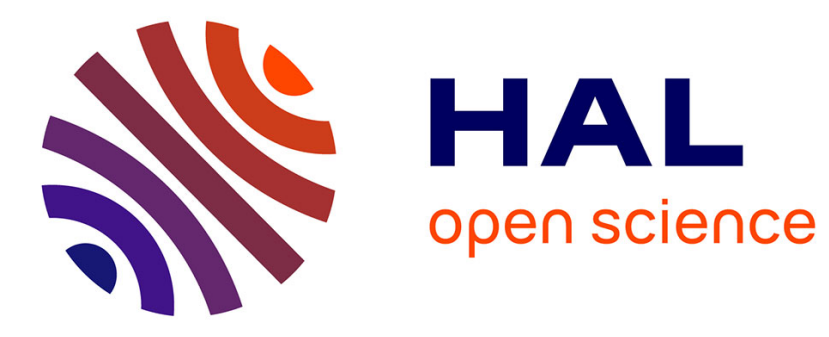

\title{
Une démarche participative pour des jeunes ayant une trisomie 21 au Togo
}

\author{
Laurence Joselin, Bernadette Céleste
}

\section{To cite this version:}

Laurence Joselin, Bernadette Céleste. Une démarche participative pour des jeunes ayant une trisomie 21 au Togo. Manon Masse, Geneviève Piérart, Francine Julien-Gauthier et Danièle Wolf (dir.). Accessibilité et participation sociale, Éditions ies, pp.81-90, 2020, 10.4000/books.ies.3822 . hal-03122124

\section{HAL Id: hal-03122124 \\ https://hal-inshea.archives-ouvertes.fr/hal-03122124}

Submitted on 23 Dec 2021

HAL is a multi-disciplinary open access archive for the deposit and dissemination of scientific research documents, whether they are published or not. The documents may come from teaching and research institutions in France or abroad, or from public or private research centers.
L'archive ouverte pluridisciplinaire HAL, est destinée au dépôt et à la diffusion de documents scientifiques de niveau recherche, publiés ou non, émanant des établissements d'enseignement et de recherche français ou étrangers, des laboratoires publics ou privés. 


\section{OpenEdition} Books

SEARCH Tout OpenEdition
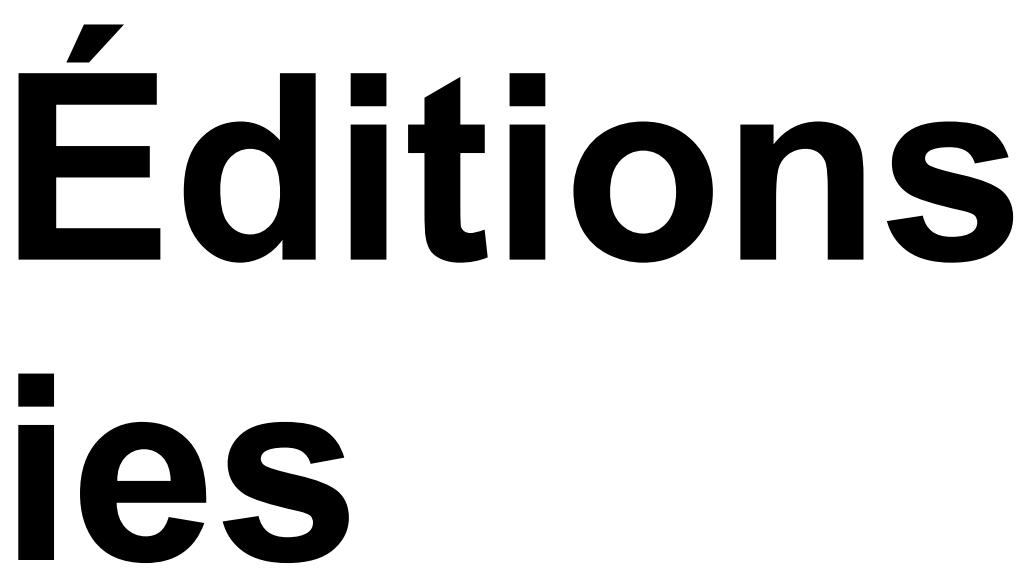

Accessibilité et participation sociale | Manon Masse, Geneviève Piérart, Francine Julien-Gauthier, et al.

Une démarche participative pour des jeunes ayant une trisomie 21 au Togo

Laurence Joselin et Bernadette Céleste

p. $81-90$

\section{Résumé}


Togo est né d'une collaboration entre professionnel-le·s paramédicaux et universitaires togolais avec une association et des chercheur.e.s français. Le partenariat a été accompli dans le cadre d'un voyage d'études à Lomé en décembre 2016. Il s'agissait d'élaborer un projet répondant aux besoins des personnes avec une trisomie 21 et de leur famille dans le contexte de la société togolaise. C'est le retour réflexif sur cette démarche qui est exposé ici.

\section{Entrées d'index}

\section{Mots clés :}

démarche participative, recherche participative, trisomie 21, Togo, accompagnement

\section{Texte intégral}

\section{La recherche participative et appliquée, de quoi s'agit-il ?}

1 La recherche participative dans le champ du handicap n'est pas un concept nouveau (Albrecht, Ravaud \& Sticker, 2001). Le mouvement des disability studies en traçait déjà les contours dans les années 1980 aux EtatsUnis, créant ainsi un champ scientifique dédié au handicap. Celui-ci ne sera pas uniquement une nouvelle discipline universitaire, mais avant tout un champ scientifique en lien avec un mouvement social militant, qui revendique la place et la visibilité des personnes en situation de handicap. "Rien sur nous sans nous » en est le slogan le plus emblématique. Dans une logique de coconstruction des savoirs, les disability studies appellent à la prise en compte de la parole des personnes concernées comme « actrices de leur devenir et de leur libération [...] considérées comme détentrices de savoirs propres que le chercheur extérieur n'a pas, même s'il peut en avoir d'autres » (Albrecht, Ravaud \& Sticker, 2001, p. 44). Toutefois,

l'originalité des disability studies n'est pas seulement dans la place, y compris scientifique, donnée aux « usagers », n'est pas simplement dans l'interdisciplinarité, n'est pas 
uniquement dans l'existence d'un mouvement de personnes handicapées, bref n'est pas dans la liste des caractéristiques que l'on peut énumérer, mais dans la rencontre, dans l'intrication de ces éléments (Albrecht, Ravaud \& Sticker, 2001, p. 45).

2 Par ailleurs, les disability studies posent en débat le statut du ou de la chercheur.e et sa légitimité, s'il n'est pas luimême en situation de handicap, dans un courant de " recherche émancipatrice ". Dans ce courant, les personnes en situation de handicap non seulement participent aux recherches, mais les contrôlent (avec un engagement politique) et les produisent (en recherchant un bénéfice immédiat pour les personnes intéressées).

Dans l'ensemble des disciplines académiques, un courant de recherche dite " participative » (ou recherche-action, recherche-intervention, recherche collaborative) se développe. Selon Couture, Bednarz et Barry (2007), celle-ci se différencie de la recherche "fondamentale » ou de laboratoire sur plusieurs points dont les principales caractéristiques sont : 1) l'articulation de la théorie et de la pratique, en associant chercheur.e.s et acteurs de terrain (associations, familles, personnes en situation de handicap) ; 2) l'implication de tous les acteurs, afin que leur voix soit entendue au même titre que la voix des chercheur·e.s ; et 3) la finalité concrète et pratique de la recherche, au bénéfice des acteurs de terrain.

Ainsi, la recherche participative permet-elle la création d'un savoir « hybride », qui n'aurait pu être construit ni par les seul·e.s chercheur.e.s ni par les seuls acteurs de terrain. La richesse des connaissances produites réside dans l'élaboration commune, dans la construction mutuelle, dans le respect et dans la reconnaissance des savoirs de chacun.e.

5 Dans notre champ, la recherche appliquée a pour but d'apporter des solutions concrètes aux difficultés rencontrées au quotidien par des personnes en situation de handicap (Benkirane \& Zouhairi (s.d.) ; Piatta, 2010). Elle doit être le fruit d'une collaboration réelle, tout au long du 
processus de recherche, entre les personnes concernées, leur famille, les accompagnant.e.s, les professionnel-le·s et les chercheur·e.s. Ses résultats doivent être diffusés aussi bien par le biais d'articles scientifiques que par des moyens susceptibles d'intéresser une diversité d'acteurs de terrain. Cette diffusion auprès du public non scientifique est indispensable pour que les connaissances produites par la recherche s'appliquent réellement et améliorent concrètement le quotidien des personnes concernées. Elle se fera dans des langages et avec des supports d'application (outils de formation, écrits adaptés, vidéos, outils opérationnels...) accessibles aux publics visés.

\section{L'objectif et le déroulement du voyage d'études}

6 Le voyage d'études s'est déroulé en plusieurs temps : entretiens auprès des familles, rencontres de partenaires institutionnels et un workshop réunissant l'ensemble des acteurs.

\section{Les acteurs du projet}

7 La demande a été initiée par des professionnel-le·s paramédicaux, membres de l'Association des parents et amis des personnes encéphalopathes (Apape), qui se sont adressés à une association française spécialisée dans ce domaine, Trisomie 21 France. L’Apape est engagée depuis plusieurs années dans un processus d'inclusion des personnes handicapées dans les communautés du Togo. Ces deux associations ont ensuite fait appel au Grhapes (Groupe de recherche sur le handicap, l'accessibilité, les pratiques éducatives et scolaires, Institut national supérieur de formation et de recherche pour l'éducation des jeunes handicapés et les enseignements adaptés) ainsi qu'à l'université de Lomé (départements de sociologie et d'anthropologie) ${ }^{1}$.

8 Tous les partenaires étaient d'accord sur la nécessité d'un travail de co-construction sans hiérarchie des statuts, tout 
comme sur l'importance cruciale d'associer au plus près les familles des personnes ayant une trisomie 21. L'objectif d'aboutir à une action concrète a rapidement déterminé le choix d'une démarche participative.

\section{Un état des lieux de la situation du terrain}

9 Cet état des lieux, réunissant professionnel-le·s et chercheur·e·s français·e.s et togolais·e·s, s'est appuyé sur des visites auprès de sept familles ainsi que sur des rencontres avec des partenaires institutionnels.

10 Les visites dans les familles ont permis aux chercheur.e.s français·e·s de découvrir le contexte local et les questions « réelles » du terrain. Ces visites organisées et réalisées par des professionnel·le·s déjà connu·e·s des familles leur ont permis de voir une diversité de situations. La confiance déjà construite entre ces professionnel-le·s et les familles, de même que leur connaissance de la langue Ewé a considérablement bénéficié aux chercheur·e·s français·e·s pour le recueil d'informations.

11 Deux visites programmées - celle du PITR/RBC (Programme des incapacités, traumatismes, préventions et réadaptation à base communautaire) et celle de la Fetaph (Fédération togolaise des associations des personnes handicapées) ont abouti à deux constats. Premièrement, un écart a été observé entre la " situation ici et maintenant » des personnes ayant une trisomie 21 et les revendications du réseau associatif, composé d'intellectuel.le·s « familles, professionnel.le.s, chercheur·e.s » qui revendiquent et partagent les valeurs de la Convention des Nations Unies relative aux droits des personnes handicapées. Par exemple, les actions en faveur de l'accessibilité de l'école (en accord avec l'article 9 de la Convention relative aux droits des personnes handicapées) portent sur l'accessibilité physique des lieux, comme l'aménagement de rampes d'accès, l'adaptation des classes (tableau plus bas, tables plus basses), et concernent essentiellement des élèves avec un handicap moteur. Les 
professionnel-le·s, les familles et les chercheur.e.s s'accordent sur l'idée d'étendre cette accessibilité physique des lieux à l'accueil d'autres élèves, y compris celles et ceux avec une trisomie 21, et revendiquent le droit à l'éducation pour tous, tel que stipulé dans l'article 24 de la convention. Deuxièmement, on constate un certain délaissement de la trisomie 21, peu connue au Togo, aux représentations diverses et fortes mais qui apparaît comme " non urgente » car elle n'est pas réputée engendrer de douleur physique particulière dans la petite enfance et ne bénéficie pas de modèle d'accompagnement, d'éducation ou de rééducation clairement identifié. Ainsi, le projet «Maman et moi sans handicap au Togo » (programme des incapacités traumatismes/prévention réadaptation, financé par Christoffel Blindenmission), entre 2015 et 2016, n’a reçu aucune demande pour un enfant ayant une trisomie 21, probablement par méconnaissance des familles des bénéfices d'une prise en charge précoce.

\section{La co-construction des connaissances}

12 Un workshop de deux jours et demi a réuni une trentaine de personnes (universitaires, membres associatifs, spécialistes de la santé et de la réadaptation, parents, représentant·e.s du ministère de l'Action sociale). Cette rencontre a permis de faire émerger les questions préoccupant les familles et les bases nécessaires aux chercheur·e·s pour élaborer leur thématique de recherche.

\section{Les apports théoriques}

13 Ces communications ont été l'occasion d'un premier croisement de regards entre les références et les connaissances des un·e.s et des autres.

14 Pour les chercheur·e.s, les questions méthodologiques ont été prioritaires. On a pu constater une référence commune (Togo, France), relative aux conditions de la recherche appliquée et aux outils déjà élaborés à cet effet au Togo. Toutefois, les méthodes de sensibilisation directe de la 
société civile restent à définir en tenant compte des réalités du contexte : culture, langue, analphabétisme, etc.

15 Les professionnel-le·s togolais ont mis l'accent sur leur malaise et la nécessité de trouver un contexte de travail unifié. Ils sont confrontés à l'articulation, voire à la contradiction de deux champs de références : celles émanant de la culture et de la tradition, fortement partagées par les familles et la société civile en général, qui situent cette pathologie dans un contexte traditionnel et mystique ; et celles diffusées sur un plan international, en référence aux connaissances scientifiques, à un développement de techniques de remédiation et aux valeurs de non-discrimination.

16 Après avoir insisté sur l'importance de la différence de contexte sociétal, les professionnel-le·s français ont présenté des connaissances sur la trisomie 21 concernant ses caractéristiques, le développement des enfants et la prise en charge précoce (Rotten, Decroix \& Levaillant, 2005 ; Trisomie 21 France, 2017). Ils ont ensuite décrit des stratégies d'accompagnement existantes pour favoriser le développement des personnes ayant une trisomie 21 et leur autodétermination, une présentation du FALC (langage facile à lire et à comprendre), ainsi que du site internet " santé très facile ». Ces connaissances sont en lien avec les valeurs développées dans la Convention des Nations Unies relative aux droits des personnes handicapées, concernant par exemple la santé (article 25) ou l'accessibilité de l'information (article 9).

\section{Le workshop}

17 De ces moments riches d'échanges et d'écoute (ateliers et groupes restreints) ont émergé quatre thématiques principales témoignant de la diversité des positionnements envers la trisomie 21 et illustrant le malaise décrit par les professionnel-le·s :

La connaissance du syndrome : globalement, la connaissance est faible et reflète le manque d'intérêt 
pour la trisomie 21. Dans la majorité des cas, rien n'a été dit aux familles à la naissance et les hypothèses explicatives de ce silence sont multiples : méconnaissance des signes cliniques par les professionnel-le·s, crainte des conséquences de ce type d'annonce sans solution à proposer, référence aux représentations traditionnelles sur l'origine de la pathologie, etc. La forme spécifique des yeux de la personne ayant une trisomie 21 amène familles et professionnel-le·s à proposer son classement dans la catégorie des déficiences visuelles. Seul.e.s les chercheur·e.s ont une connaissance médicale, sociale ou psychologique de la pathologie.

L'origine de la pathologie et les représentations : la diversité de situations décrites par les familles et les professionnel-le·s reflète l'hétérogénéité des représentations (Mbassa Menick, 2015 ; Dassa, Mbassa Menick, Tabo, Ahyi \& Ferrari, 2009) concernant l'origine du syndrome. Aucune famille n'a évoqué l'origine génétique de la trisomie. Des représentations sociales communément partagées par la population sont évoquées : références à l'enfant serpent, aux sorciers, aux divinités maléfiques aux pouvoirs surnaturels, susceptibles de jeter un sort « qui ferait devenir pareil ». Pour les professionnel-le·s et chercheur·e·s, la référence à une origine génétique (restant floue au demeurant) est partagée. Les professionnel-le·s et chercheur.e.s connaissent les représentations traditionnelles et leur persistance dans la population est citée comme l'obstacle principal à l'évolution des pratiques à l'égard des personnes ayant une trisomie 21.

La participation sociale : selon les milieux ou les quartiers, la participation sociale est décrite par les personnes consultées comme étant réalisée (exemple : jeune homme apprenti coiffeur) ou impossible (exemple : enfermement d'une jeune femme par crainte d'agression sexuelle). Les professionnel-le·s 
connaissent bien les situations individuelles de ces jeunes, mais leur action est essentiellement orientée vers le soutien à la scolarisation des enfants. Les conditions de scolarisation sont toutefois telles (effectifs des classes, manque de formation des enseignants) que les effets de cette participation sociale sont difficilement constatables.

Les conséquences économiques de la pathologie : les difficultés matérielles sont en tête des difficultés évoquées par les familles et elles sont accentuées par le fait d'avoir un enfant avec une trisomie 21 (exemple : mère se retrouvant seule sitôt reconnue la spécificité de l'enfant, abandon par le père). Le suivi médical et l'éducation demandent un investissement financier hors de portée de ces mères. Les professionnel-le·s tentent de pallier cette situation en créant des espaces permettant d'accompagner les mères dans une activité génératrice de revenus. Le modèle économique est celui du microcrédit. C'est aussi autour de cette thématique que se sont rencontrés chercheur·e·s et professionnel-le·s, car les chercheur·e.s sociologues présent.e.s avaient déjà réalisé une étude sur la possibilité d'accès au crédit des femmes handicapées et des mères des enfants handicapés (Agbovi, 2010).

\section{Le choix de la thématique de recherche}

18 Les questions liées à l'apprentissage des jeunes ayant une trisomie 21 et à leur insertion professionnelle répondent à une double préoccupation : assurer leur autonomie financière et soulager leur famille, et permettre leur « survie » après le décès de la mère ou des parents.

19 Il pourrait être judicieux de s'appuyer sur l'expérience du microcrédit, déjà mise en œuvre, que les familles et professionnel-le·s se sont appropriée et qui donne des résultats positifs évalués par la recherche. Pourquoi ne pas concevoir un système de microcrédit impliquant aussi les jeunes adultes avec une trisomie 21, et pas uniquement 
leur mère ? Cela pourrait constituer une piste de réflexion et de recherche pour opérationnaliser la question de l'avenir professionnel de ces jeunes.

\section{Conclusion}

20 L'expérience de ce voyage d'études au Togo a permis d'éprouver, au-delà du souhait commun de la mise en œuvre d'une démarche participative, quelques limites à sa réalisation immédiate ; la concertation et la consultation des acteurs principalement intéressés ne posent pas de problème particulier, mais l'étape de la codécision n'est pas totalement aboutie.

21 Si la participation des principaux intéressés est requise pour élaborer une véritable démarche participative, les familles rencontrées n'ont pas encore une posture suffisamment solide pour interagir d'égal à égal avec des

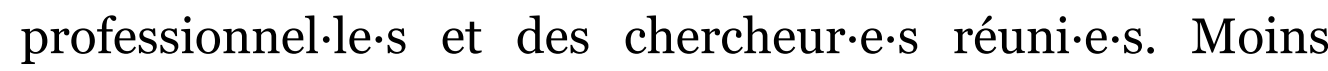
diplômées, de classe sociale défavorisée, sans la maîtrise de la langue française (parlée par les professionnel·le·s et les chercheur·e.s togolais), il n'est pas évident pour ces personnes d'affirmer leur point de vue face aux chercheur·e.s. C'est pourquoi il apparaît nécessaire de s'appuyer, durant une période de transition, sur les professionnel-le·s qui bénéficient, tant du côté des familles que des chercheur.e.s, d'une extrême confiance et considération pour le travail accompli sur le terrain. Ces professionnel-le·s pourront recueillir le point de vue des familles et le partager, en attendant que celles-ci acquièrent plus d'assurance, davantage d'habitude de participation, pour ensuite prendre elles-mêmes la parole. Cette démarche pourrait mener à la mise en place des conditions nécessaires à une recherche participative.

22 Les différences liées à la culture : les rapprochements sont relativement aisés entre les statuts ou positionnements en ce qui concerne les chercheur·e·s et les professionnel.le·s. Togolais ou Français, professionnel-le.s et chercheur.e.s sont souvent issu·e·s des mêmes domaines de formation, 
partagent les mêmes champs théoriques et se retrouvent sur les mêmes thématiques (exemple : l'apprentissage, l'orthophonie ou la trisomie 21 sont étudiés en santé, en éducation et en réadaptation). Aussi partagent-ils plus ou moins les mêmes représentations de la trisomie, de son origine ou de ses conséquences. Les questionnements des familles sont apparus comme ancrés dans un contexte symbolique et social qui diffère des représentations des chercheur·e.s et professionnel-le·s, y compris celles et ceux d'origine togolaise. Si ces derniers connaissent le socle symbolique des représentations des familles (enfant serpent, divinité maléfique), ils affirment ne pas le partager, bien que vivant dans le même contexte culturel.

23 La difficulté du recueil de la parole des personnes principalement intéressées, lorsqu'elles n'ont pas la possibilité ou éprouvent de grandes difficultés à s'exprimer par elles-mêmes, peut contredire le principe de la participation active de ces acteurs à la recherche. Ainsi, le dialogue avec les familles a été rendu possible grâce à un interprétariat (ce qui constitue déjà un biais par rapport à la rencontre directe). D'autre part, les échanges avec certains jeunes ayant une trisomie 21 (les plus jeunes, les plus timides, ou ceux qui parlent peu, difficilement, ou pas du tout) se sont avérés plus difficiles, voire impossibles. En réponse à cette dernière difficulté, plus classiquement, les parents ont été interrogés, aux côtés de leur enfant ayant une trisomie 21. Toutefois, une méthodologie de recueil direct de la parole des jeunes ayant une trisomie 21 pourrait constituer l'un des axes de prolongement de ce voyage d'études, dans la continuité des travaux émergents sur la participation la plus directe possible des personnes dont la communication est entravée (Bedoin \& Scelles, 2015).

24 Une autre difficulté, critique déjà adressée de façon légitime aux disability studies (Albrecht, Ravaud \& Sticker, 2001), concerne la portée et la faisabilité de la recherche participative dans un pays où les priorités des personnes concernées sont orientées par des besoins 
fondamentaux et non par des questions de démarche participative. Il faut sans nul doute un certain recul, une certaine sécurité et quiétude pour poser un regard réflexif et pouvoir analyser sa propre situation en traduisant ses difficultés en questionnements. Aussi, s'il est évident que toutes les familles n'ont pas la même implication dans l'élaboration de la démarche, des protocoles élaborés conjointement doivent garantir qu'elles seront écoutées, consultées et intéressées aux résultats qui leur reviendront en premier lieu.

25 L'expérience relatée ici ne constitue pas un « modèle à suivre »; il s'agit avant tout du témoignage d'une démarche collective tentant de répondre au mieux, du côté de la recherche comme de celui des praticien·ne.s, à une demande sociale de terrain impliquant les personnes ayant une trisomie 21.

26 Même s'il est difficilement possible de résoudre intégralement ces limites à la participation pleine et entière de l'ensemble des acteurs concernés, prendre conscience de ces restrictions permet de tenter d'en amoindrir les impacts. En tout état de cause, la recherche participative ne saurait être envisageable sans une implication égale des acteurs, avec l'objectif de répondre à des priorités utiles et concrètes qui ont du sens pour les personnes concernées. C'est dans ce lien que la participation des acteurs de terrain pourra se mettre en œuvre. Celle-ci impose également de rester humble jusqu'au bout... et d'accepter d'écouter, de considérer, de respecter, totalement, des personnes qui n'ont pas l'habitude de parler et de mettre en mots leur situation.

\section{Bibliographie}

Des DOI sont automatiquement ajoutés aux références par Bilbo, l'outil d'annotation bibliographique d'OpenEdition.

Les utilisateurs des institutions qui sont abonnées à un des programmes freemium d'OpenEdition peuvent télécharger les références bibliographiques pour lequelles Bilbo a 


\section{trouvé un DOI.}

Cette bibliographie est disponible grâce à la souscription de votre institution à un des programmes freemium d'OpenEdition. Elle contient toutes les références automatiquement générées par Bilbo en utilisant Crossref.

Format

APA

MLA

Chicago

Albrecht, G. L., Ravaud, J.-F., \& Stiker, H.-J. (2001). L'émergence des disability studies : état des lieux et perspectives. Sciences sociales et santé, 19(4), 43-73. https://doi.org/10.3406/sosan.2001.1535

Mbassa Menick, D. (2015). Les représentations sociales et culturelles du handicap de l'enfant en Afrique noire. Perspectives Psy, 54(1), 30-43. https://doi.org/10.1051 /ppsy/2015541030

Albrecht, G. L., J.-F. Ravaud, and H.-J. Stiker. "L'émergence des disability studies : état des lieux et perspectives”. Sciences sociales et santé, vol. 19, no. 4, PERSEE Program, 2001, pp. 43-73. Crossref, doi:10.3406/sosan.2001.1535.

Mbassa Menick, D. "Les représentations Sociales Et Culturelles Du Handicap De l'enfant En Afrique Noire". Perspectives Psy, vol. 54, no. 1, EDP Sciences, Aug. 2015, pp. 30-43. Crossref, doi:10.1051/ppsy/2015541030.

Albrecht, Gary L., J.-F. Ravaud, and Henri-Jacques Stiker. "L'émergence des disability studies : état des lieux et perspectives". Sciences sociales et santé 19, no. 4 (2001): 43-73. https://doi.org/10.3406/sosan.2001.1535.

Mbassa Menick, Daniel. "Les représentations Sociales Et Culturelles Du Handicap De l'enfant En Afrique Noire”. Perspectives Psy 54, no. 1 (August 2015): 30-43. https://doi.org/10.1051/ppsy/2015541030.

Agbovi, K. (2010). Représentations et perception du handicap par les cadres de l'administration publique et les autorités locales : Bénin, Burkina Faso, Mali, Niger, 
Sénégal, Sierra Leone, Togo. Dakar : Handicap International, coordination régionale Decisiph.

Format

APA

MLA

Chicago

Albrecht, G. L., Ravaud, J.-F., \& Stiker, H.-J. (2001). L'émergence des disability studies : état des lieux et perspectives. Sciences sociales et santé, 19(4), 43-73. https://doi.org/10.3406/sosan.2001.1535

Albrecht, G. L., J.-F. Ravaud, and H.-J. Stiker. "L'émergence des disability studies : état des lieux et perspectives". Sciences sociales et santé, vol. 19, no. 4, PERSEE Program, 2001, pp. 43-73. Crossref, doi:10.3406/sosan.2001.1535.

Albrecht, Gary L., J.-F. Ravaud, and Henri-Jacques Stiker. "L'émergence des disability studies : état des lieux et perspectives”. Sciences sociales et santé 19, no. 4 (2001): 43-73. https://doi.org/10.3406/sosan.2001.1535.

Cette référence bibliographique est disponible grâce à la souscription de votre institution à un des programmes freemium d'OpenEdition.

Elle a été automatiquement générée par Bilbo en utilisant Crossref.

Albrecht, G., Ravaud, J.-F. \& Sticker, H. J. (2001). L'émergence des disability studies : état des lieux et perspectives. Sciences sociales et santé, 19 (4), 43-73.

DOI : 10.3406/sosan.2001.1535

Bedoin, D. \& Scelles, R. (2015). S'exprimer et se faire comprendre. Entretiens et situations de handicap. Toulouse : Erès.

Benkirane, W. \& Zouhairi, A. (s.d.). La recherche appliquée sur le handicap en Afrique. Mapping Maghreb et Afrique de l'Ouest. Paris : Firah. 
Couture, C., Bednarz, N. \& Barry, S. (2007). Multiples regards sur la recherche participative. Dans M. Anadon (Dir.), La recherche participative (pp. 217-234). Québec : Presses de l'Université du Québec.

Dassa, S. K., Mbassa Menick, D., Tabo, A., Ahyi, R. G. \& Ferrari, P. (2009). Les perceptions collectives des enfants et adolescents handicapés mentaux en Afrique de l'Ouest. Le cas du Togo. Neuropsychiatrie de l'enfance et de l'adolescence, 57 (4), 325-331.

Format

APA

MLA

Chicago

Mbassa Menick, D. (2015). Les représentations sociales et culturelles du handicap de l'enfant en Afrique noire. Perspectives Psy, 54(1), 30-43. https://doi.org/10.1051 /ppsy/2015541030

Mbassa Menick, D. "Les représentations Sociales Et Culturelles Du Handicap De l'enfant En Afrique Noire”. Perspectives Psy, vol. 54, no. 1, EDP Sciences, Aug. 2015, pp. 30-43. Crossref, doi:10.1051/ppsy/2015541030.

Mbassa Menick, Daniel. "Les représentations Sociales Et Culturelles Du Handicap De l'enfant En Afrique Noire”. Perspectives Psy 54, no. 1 (August 2015): 30-43. https://doi.org/10.1051/ppsy/2015541030.

Cette référence bibliographique est disponible grâce à la souscription de votre institution à un des programmes freemium d'OpenEdition.

Elle a été automatiquement générée par Bilbo en utilisant Crossref.

Mbassa Menick, D. (2015). Les représentations sociales et culturelles du handicap de l'enfant en Afrique noire. Perspectives Psy, 54 (1), 30-43.

DOI : $10.1051 / \mathrm{ppsy} / 2015541030$

Piatta, F. (2010). Les droits en actions. Bonnes pratiques 
pour une gouvernance locale inclusive en Afrique de l'Ouest : Bénin, Burkina Faso, Mali, Niger, Sénégal, Sierra Leone, Togo. Dakar : Handicap International, coordination régionale Decisiph.

Rotten, D., Decroix, H. \& Levaillant, J. M. (2005). La trisomie 21 : prise en charge, du diagnostic anténatal à l'adolescence. Paris : EDK.

Trisomie 21 France. (2017). 100 idées pour en savoir plus sur les personnes avec trisomie 21... et casser les idées reçues. Paris : Tom Pousse.

\section{Notes}

1. Le voyage d'études a été soutenu par la Firah (Fondation internationale de la recherche appliquée sur le handicap). Il a été mis en œuvre au Togo notamment par Vincent-Irénée Agbovi (Université de Lomé), Victor Alipui (Apape), Atiyihwè Awesso (Université de Lomé), Daniel K. Dahouindji (Apape), Yvette Monkam (Lomé), Gbati Napo (Université de Lomé) et Frédéric Tagba (Centre national d'appareillage orthopédique et de rééducation fonctionnelle).

\section{Auteurs}

\section{Laurence Joselin}

\section{Docteure en psychologie et} ingénieure de recherche au Grhapes (Groupe de recherche sur le handicap, l'accessibilité, les pratiques éducatives

et scolaires) ; ses travaux portent sur la représentation sociale du handicap dans la littérature de jeunesse et les questions de 


\section{parentalité en situation de handicap.}

\section{Bernadette Céleste}

Docteure en psychobiologie génétique et comparative, elle est membre de Trisomie 21 France et ex-directrice de l'Institut national de formation et de recherche sur l'éducation des jeunes handicapés et les enseignements adaptés.

(C) Éditions ies, 2020

Conditions d'utilisation : http://www.openedition.org/6540

Référence électronique du chapitre

JOSELIN, Laurence ; CÉLESTE, Bernadette. Une démarche participative pour des jeunes ayant une trisomie 21 au Togo In : Accessibilité et participation sociale : Vers une mise en œuvre de la Convention relative aux droits des personnes handicapées [en ligne]. Genève : Éditions ies, 2020 (généré le 23 décembre 2021). Disponible sur Internet : <http://books.openedition.org/ies/3822>. ISBN : 9782882242211. DOI : https://doi.org/10.400o/books.ies.3822.

Référence électronique du livre

MASSE, Manon (dir.) ; et al. Accessibilité et participation sociale : Vers une mise en ouvre de la Convention relative aux droits des personnes handicapées. Nouvelle édition [en ligne]. Genève : Éditions ies, 2020 (généré le 23 décembre 2021). Disponible sur Internet : <http://books.openedition.org/ies/3697>. ISBN : 9782882242211. DOI : https://doi.org/10.400o/books.ies.3697.

Compatible avec Zotero 